Bull. Austral. Math. Soc.

VOL. 55 (1997) [327-333]

\title{
HOMOLOGICAL PROPERTIES OF SF RINGS
}

\author{
Ahmad Shamsuddin
}

\begin{abstract}
A ring $R$ is said to be left $S F$ if all simple left $R$-modules are flat. We study some homological properties of such rings and find situations in which these become von Neumann regular.
\end{abstract}

\section{INTRODUCTION}

A ring $R$ is said to be left $S F$ if all simple left $R$-modules are flat. It has been conjectured by Ramamurthi [9] that such rings are necessarily von Neumann regular. This conjecture has been verified in a number of situations-see, for example, $[\mathbf{3}, \mathbf{6}$, $8,9,12,13,14]$, and no counterexample to it is presently known. The purpose of this note is to study some homological properties of such rings and discuss situations in which this conjecture is true. Thus we show that if all the principal right ideals of a left SF ring $R$ are projective, then $R$ is von Neumann regular. And when a left SF ring $R$ has weak global dimension at most 1 and is right coherent, we show that $R$ is von Neumann regular.

Next we turn to discuss the question of the vanishing of the Jacobson radical of a left SF ring $R$. We prove that if any power $J^{n}$ of the radical $J$ is finitely generated as a left ideal of the ring, then $J^{n}=0$. If $R$ is left self-injective and $J_{R}$ is pseudo-coherent, then $R$ is von Neumann regular.

It was shown in Theorem 1.3 of [13] that if a left SF ring satisfies ACC on principal left ideals then $R$ is semi-simple Artinian. We prove, more generally, that if $R$ is a subring of a ring all of whose cyclic flat left modules are projective, then $R$ is semisimple artinian.

Throughout the present note, the right $R$-module $M_{R}^{+}=\operatorname{Hom}_{Z}(M, \mathbb{Q} / \mathbb{Z})$ will denote the character module of the left $R$-module ${ }_{R} M$, and $\operatorname{rad}\left({ }_{R} M\right)$ will denote the Jacobson radical of the left $R$-module $M$. The singular submodule of a module ${ }_{R} M$ is denoted by $Z\left({ }_{R} M\right)$. Thus, $Z\left({ }_{R} R\right)$ stands for the left singular ideal of $R$, which is a two-sided ideal of $R$. We write $J$ for the radical of the ring itself. The right annihilator (respectively left annihilator) of an element $x \in R$ is denoted by $r(x)$ (respectively $\ell(x))$. wd $M$ stands for the weak dimension of $M$.

Received 1st July, 1996

Copyright Clearance Centre, Inc. Serial-fee code: 0004-9729/97 \$A2.00+0.00. 


\section{Homological Properties of SF Rings}

A ring $R$ is said to be right Rickart if for every $x \in R$, the right ideal $r(x)$ is generated by an idempotent, or equivalently, $x R$ is projective.

Theorem 2.1. A left SF right Rickart ring is von Neumann regular.

Proof: Let $x \in R$ and let $\operatorname{rad} R / R x=L / R x$. Put $r(x)=e R$ where $e=e^{2}$ and set $f=1-e$. If $I$ is a maximal left ideal of $R$ and $x \in I$ then $x=x a$ for some $a \in I$ because $R / I$ is a flat left $R$-module. Hence $1-a \in r(x)$ and so $f=f a \in I$. This implies that $f \in L$. Note that

$$
R / R x=(R e+R x) / R x \oplus(R f+R x) / R x,
$$

so because $(R f+R x) / R x$ is superfluous in $R / R x$, we see that $R=R e+R x$. Since $x e=0$, we see that, indeed, $R=R e \oplus R x$. Hence all principal left ideals of $R$ are direct summands of $R$, whence $R$ is von Neumann regular.

Recall that a module ${ }_{R} M$ is said to be pseudo-coherent if each of its finitely generated submodules is finitely presented. A finitely generated pseudo-coherent module is said to be coherent. If ${ }_{R} R$ is coherent, then we say that $R$ is left coherent. It is well-known that a ring $R$ is left coherent if and only if the direct product of every family of flat right $R$-modules is flat; see, for example, [1, Theorem 19.20]. Recall also that the weak global dimension of a ring is at most 1 if and only if all submodules of flat modules are flat.

A submodule $A$ of a module ${ }_{R} B$ is said to be pure in $B$ if for every right $R$-module $P$, the homomorphism $P \otimes A \rightarrow P \otimes B$ is injective. An epimorphism $f: A \rightarrow B$ is said to be pure if $\operatorname{Ker} f$ is pure in $A$. It is well-known that epimorphisms onto flat modules are pure (see, for example, [2, Proposition 4, p.15]), and a finitely presented module $M$ is pure projective in the sense that for every pure epimorphism $f: A \rightarrow B$, every homomorphism $g: M \rightarrow B$ lifts to a homomorphism $g: M \rightarrow A$; see [11].

LEMMA 2.2. Let $R$ be a semi-primitive ring and let ${ }_{R} M$ be a finitely presented module such that $M / \operatorname{rad} M$ is flat. Then $M$ is projective.

Proof: Consider the pure exact sequence

$$
0 \longrightarrow \operatorname{Ker} f \longrightarrow F \stackrel{f}{\longrightarrow} M / \operatorname{rad} M \longrightarrow 0
$$

where $F$ is a free $R$-module. Since $M$ is pure projective, there is $\theta: M \rightarrow F$ such that $f \theta=\pi$, where $\pi: M \rightarrow M / \operatorname{rad} M$ is the canonical projection. It follows that $F=K+\operatorname{Im} \theta$ where $K=\operatorname{Ker} f$ and it is easy to verify that $K \cap \operatorname{Im} \theta=0$ because $\theta(\operatorname{rad} M) \subseteq \operatorname{rad} F=0$. Hence $F=K \oplus \operatorname{Im} \theta$ and so $M / \operatorname{rad} M \cong F / K \cong \operatorname{Im} \theta$ is projective. This shows that $\operatorname{rad} M=0$ and so $M$ is projective. 
Proposition 2.3. A right coherent left SF ring with weak global dimension $\leqslant 1$ is von Neumann regular.

Proof: Suppose $M$ is a finitely presented left $R$-module. It follows from [1, Proposition 9.16$]$ that $M / \operatorname{rad} M$ embeds in a direct product of flat modules, whence embeds in a flat module, so it is necessarily flat. In particular, $R / J$ is a flat $R$-module so that $J=0$. It follows from Lemma 2.2 that all finitely presented modules are projective. This implies that $R$ is von Neumann regular.

\section{THE JACOBSON RADICAL OF A LEFT SF RING}

If $M$ is a left $R$-module, we shall write $M^{*}$ for the right $R$-module $\operatorname{Hom}_{R}(M, R)$. The trace of $M$ is the two-sided ideal $T(M)$ defined as $\sum_{f \in M^{*}} \operatorname{Im} f$. The module $M$ is a generator if and only if $T(M)=R$.

Proposition 3.1. Suppose that $R$ is a left $S F$ ring. Let ${ }_{R} M$ be a finitely presented module with $\operatorname{Hom}_{R}(M, R / I) \neq 0$ for a maximal left ideal $I$ of $R$. Then $T(M) \nsubseteq I$.

Proof: Let $f: M \rightarrow R / I$ be a non-zero homomorphism and denote by $\pi$ : $R \rightarrow R / I$ the canonical map. Because $M$ is pure projective, there is a homomorphism $g: M \rightarrow R$ such that $\pi \circ g=f$. This shows that $R=\operatorname{Im}(g)+I=T(M)+I$ so that $T(M) \nsubseteq I$.

Corollary 3.2. Suppose that $R$ is a left $S F$ ring. If $M$ is a finitely presented module such that $\operatorname{Hom}_{R}(M, R / I) \neq 0$ for every maximal left ideal $I$ of $R$, then $M$ is a generator. Hence with $\Lambda=$ End $_{R} M, R \cong$ End $M_{\Lambda}$.

Corollary 3.3. The Jacobson radical of a left SF ring cannot contain a nonzero two-sided ideal, finitely generated as a left ideal. In particular, if $J^{n} \neq 0$ then $J^{n}$ and $Z\left(R_{R}\right)$ are not finitely generated as left ideals.

Corollary 3.4. If $R$ is a simple left SF ring, then every non-zero finitely presented module is a generator.

Proposition 3.5. If $R$ is left $S F$ and $J$ is finitely generated and projective as a right ideal, then $J=0$.

Proof: Let $\left(I_{\lambda}\right)_{\lambda \in \Lambda}$ denote the set of all maximal left ideals of $R$. Since $R / I_{\lambda}$ is flat, $J I_{\lambda}=J \cap I_{\lambda}=J$ for each $\lambda \in \Lambda$. As $J_{R}$ is finitely generated projective, $J^{2}=J \cdot \bigcap I_{\lambda}=\bigcap J I_{\lambda}=J$, whence $J=0$ by Nakayama's lemma.

Next, we consider rings $R$ for which $R / J$ is von Neumann regular, for example, left self-injective rings. We need the following result, taken from Morita [7, Lemma 1.5]. 
LEMMA 3.6. Let $R$ be a ring and ${ }_{R} M$ an $R$-module. If $P$ is the annihilator of $M$ in $R$ then ${ }_{R} M$ is flat if and only if ${ }_{R / P} M$ is flat and for all $x \in P, 0=x R \otimes_{R} M$.

THEOREM 3.7. Let $R$ be a ring for which $R / J$ is von Neumann regular and $J_{R}$ is pseudo-coherent. If $R$ is left $S F$ then $R$ is von Neumann regular.

Proof: Let $\left(I_{\lambda}\right)_{\lambda \in \Lambda}$ denote the set of all maximal left ideals of $R$ and let $M_{\lambda}=$ $R / I_{\lambda}$. Put $M=\prod_{\lambda \in \Lambda} M_{\lambda}$, so $J$ is the annihilator of $M$ in $R$. To show that ${ }_{R} M$ is flat, it suffices to show that $x R \otimes_{R} M=0$ for all $x \in J$. But this is true because $x R$ is finitely presented and so $x R \otimes_{R} M \cong \prod_{\lambda \in \Lambda} x R \otimes_{R} M_{\lambda} \cong \prod_{\lambda \in \Lambda} x M_{\lambda}=0$. The sequence

$$
0 \rightarrow R / J \rightarrow \prod_{\lambda \in \Lambda} M_{\lambda} \rightarrow Q \rightarrow 0
$$

(where $Q=\operatorname{Coker} R / J \rightarrow \prod_{\lambda \in \Lambda} M_{\lambda}$ ) is pure in $R / J$-mod, so it is pure in $R$-mod. Hence $R / J$ is a flat left $R$-module and so $J=0$, which proves the result.

\section{REMARKS}

1. Subrings of CFP RINGs. We say that a ring $R$ is left $C F P$ if every cyclic flat left $R$-module is projective. We make the following observation.

Proposition 4.1. A left SF subring $R$ of a left CFP ring $S$ is semi-simple Artinian.

Proof: Let $M$ be a simple left $R$-module. Then $S \otimes_{R} M$ is a cyclic flat $S$ module, whence it is projective. It now follows from Jøndrup [5, Theorem 3.1] that ${ }_{R} M$ is projective, whence $R$ is semi-simple Artinian.

It is possible now to generalise Theorem 3.1 of [13], in the following fashion.

TheOREM 4.2. Let $R$ be a left $S F$ subring of a ring $S$. Then $R$ is semi-simple Artinian if

(a) $S$ is semi-perfect, or

(b) $S$ is Morita equivalent to a domain, or

(c) every ascending chain of principal left ideals of $S$ terminates, or

(d) every descending chain $r\left(x_{1}\right) \supseteq r\left(x_{2}\right) \supseteq \ldots$ terminates, or

(e) every ascending chain $\ell\left(x_{1}\right) \subseteq \ell\left(x_{2}\right) \subseteq \ldots$ terminates.

Proof: In each case, $S$ is left CFP. Specifically, (a) follows from the well-known fact that semi-perfect rings are characterised by the statement that every finitely generated module has a projective cover, and from the fact that every projective cover of a flat module is an isomorphism. To see (b), we note that Jøndrup [5] shows that for 
$n$-firs, every $n$-generated flat module is projective. In particular, any 1 -fir (that is, a domain) is left CFP, hence so is also any ring Morita equivalent to it. Note that (c) follows also from [5] as well. Now the same proof used in [13, Theorem 3.1] to show $(5) \Rightarrow(1)$ and $(6) \Rightarrow(1)$ in this reference can be used to prove that, if $S$ satisfies either of the conditions in (d) or (e), then it is left CFP. The result now follows from the previous proposition.

2. LeFT SEMI-ARTINIAN LEFT SF RINGS. It was observed in [3] that for a left semiArtinian ring (that is, every non-zero left $R$-module has a simple submodule), the weak dimension of a module $M_{R}$ can be computed as

$\operatorname{wd} M_{R}=\sup \left\{n \mid \exists\right.$ a simple module ${ }_{R} X$ such that $\left.\operatorname{Tor}_{n}^{R}(M, X) \neq 0\right\}$.

This result had already been obtained in [8], and it immediately implies that a left SF left semi-artinian ring is von Neumann regular.

3. Subrings of matrix RINGs. Let $V$ be a vector space over a field $F$ of countable dimension. We ask whether left SF subrings of $\operatorname{End}_{F} V$ are von Neumann regular. The following observation is useful in determining that certain known subrings of this ring are not SF, hence not von Neumann regular.

Proposition 4.3. Suppose that $R$ is left $S F$. If $R$ is a subring of a ring $S$, and if $x \in R$ is left invertible in $S$, then it is left invertible in $R$.

Proof: If $R x \neq R$, then $R x \subseteq I$ for some maximal left ideal $I$ of $R$. Then $x=x a$ for some $a \in I$. If $y x=1$ for some $y \in S$, then $a=1$, so $I=R$. This is is a contradiction.

Call an element $f \in \operatorname{End}_{F} V$ locally nilpotent if $V=\bigcup_{n=1}^{\infty} \operatorname{Ker} f^{n}$. If $f$ is locally nilpotent, then $1-f$ is invertible, with inverse $1+f+f^{2}+\ldots$. For example, if $V$ has a basis $\left\{e_{1}, e_{2}, \ldots\right\}$, then the linear transformation given by

$$
f\left(e_{1}\right)=0, \quad f\left(e_{i}\right)=e_{i-1} \quad \text { if } i>1
$$

is locally nilpotent. Denote by $B(F)$ the ring of all $\aleph_{0} \times \aleph_{0}$ matrices with entries in a field having only finitely many non-zero entries in each row and column. Since $B(F)$ contains $f$ defined by $(*)$, it is not left SF. Similarly, the ring constructed by Tjukavkin in $[10]$ is not left $\mathrm{SF}$.

4. FP-Injective RINGs. Recall that a module ${ }_{R} M$ is said to be $F P$-injective if for every finitely presented module ${ }_{R} F, \operatorname{Ext}_{R}^{1}(F, M)=0$. The ring $R$ is said to be left $F P$-injective if ${ }_{R} R$ is FP-injective. For example, all von Neumann regular rings are right and left FP-injective. We have 
PROPOSITION 4.4. Suppose that $R$ is a left SF ring for which every finitely presented module has zero radical. Then $R$ is left FP-injective.

Proof: Suppose $P$ is a finitely presented module. Let $\left(I_{\lambda}\right)_{\lambda \in \Lambda}$ denote the set of all maximal left ideals of $R$. Then we have a commutative diagram

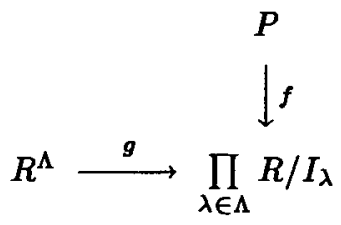

where $f$ is injective and $g$ is an epimorphism. Since the direct product of pure epimorphisms is also a pure epimorphism, it follows that $g$ is a pure epimorphism. Since $P$ is pure projective, there exists a homomorphism $h: P \rightarrow R^{\Lambda}$ such that $g h=f$. Now $h$ is necessarily injective, so $P$ is torsionless. Thus all finitely presented left $R$-modules are torsionless, whence $R$ is right FP-injective, by [4, Theorem 2.3].

If ${ }_{R} A$ and ${ }_{R} B$ are modules such that ${ }_{R} A$ is finitely presented, then we have an epimorphism

$$
\operatorname{Tor}\left(B^{+}, A\right) \rightarrow \operatorname{Ext}(A, B)^{+}
$$

which is an isomorphism if $R$ is left coherent. It follows that if $M_{R}^{+}$is flat then ${ }_{R} M$ is left FP-injective. If $R$ is left SF then we can say more. In this case, if ${ }_{R} S$ is any simple module, then $S^{+}$is a direct summand of $R^{+}$, so $S_{R}^{+}$is flat and hence (†) implies that ${ }_{R} S$ is FP-injective. In particular, this implies that $R$ is semi-primitive. To see this, suppose $A$ is a non-zero superfluous finitely generated left ideal of $R$. Then ${ }_{R} A$ has a maximal submodule ${ }_{R} B$, whence we have an exact sequence

$$
0 \rightarrow A / B \rightarrow R / B \rightarrow R / A \rightarrow 0
$$

with $R / A$ finitely presented and $A / B$ simple. The sequence splits because $\operatorname{Ext}(R / A, A / B)=0$, in contradiction with the assumption that $A$ is superfluous in $R$ (so that $A / B$ is superfluous in $R / B$.) This proves that $\operatorname{rad} R=0$.

\section{REFERENCES}

[1] F.W. Anderson and K.R. Fuller, Rings and categories of modules (2nd edition), Graduate texts in mathematics (Springer-Verlag, Berlin, Heidelberg, New York, 1992).

[2] N. Bourbaki, Elements of mathematics-commutative algebra, (Chapters 1-7) (SpringerVerlag, Berlin, Heidelberg, New York, 1989).

[3] Nanqing Ding and Jianlong Chen, 'The homological dimensions of simple modules', Bull. Austral. Math. Soc. 48 (1993), 265-274. 
[4] S. Jain, 'Flat and FP-injectivity', Proc. Amer. Math. Soc. 41 (1973), 437-442.

[5] S. Jøndrup, 'On finitely generated flat modules II', Math. Scand. 27 (1970), 105-112.

[6] R. Yue Chi Ming, 'On von Neuman regular rings-II', Math. Scand. 39 (1976), 167-170.

[7] K. Morita, 'Flat modules, injective modules and quotient rings', Math. Z. 120 (1971), 25-40.

[8] C. Nšatăsescu, 'Dimension globale des anneaux semi-artiniens', C.R. Acad. Sci. Paris Sér. $A-B 268$ (1969), A685-688.

[9] V.S. Ramamurthi, 'On the injectivity and flatness of certain cyclic modules', Proc Amer. Math. Soc. 48 (1975), 21-25.

[10] D.V. Tjukavkin, 'Rings all of whose one sided ideals are generated by idempotents', Comm. Algebra 17 (1989), 1193-1198.

[11] R.B. Warfield, 'Purity and algebraic compactness for modules', Pacific J. Math. 28 (1969), 699-719.

[12] Y. Xiao, 'SF rings and excellent extensions', Canad. Math. Bull. 37 (1994), 2463-2471.

[13] Y. Xiao, 'One sided SF rings with certain chain conditions', Comm. Algebra 22 (1994), 272-277.

[14] Jule Zhang and Du Xianneng, 'von Neumann regularity of SF rings', Comm. Algebra 21 (1993), 2445-2451.

Department of Mathematics

American University of Beirut

Beirut

Lebanon

e-mail: ahmad@layla.aub.edu.lb 